\title{
Bulk local states and crosscaps in holographic CFT
}

\author{
Yu Nakayama ${ }^{a, b}$ and Hirosi Ooguri ${ }^{b, c, d}$ \\ ${ }^{a}$ Department of Physics, Rikkyo University, \\ Toshima, Tokyo 175-8501, Japan \\ ${ }^{b}$ Kavli Institute for the Physics and Mathematics of the Universe (WPI), University of Tokyo, \\ Kashiwa, Chiba 27\%-8583, Japan \\ ${ }^{c}$ Walter Burke Institute for Theoretical Physics, California Institute of Technology, \\ Pasadena, CA 91125, U.S.A. \\ ${ }^{d}$ Center for Mathematical Sciences and Applications and \\ Center for the Fundamental Laws of Nature, Harvard University, \\ Cambridge, MA 02138, U.S.A. \\ E-mail: nakayama@theory.caltech.edu, ooguri@caltech.edu
}

ABSTRACT: In a weakly coupled gravity theory in the anti-de Sitter space, local states in the bulk are linear superpositions of Ishibashi states for a crosscap in the dual conformal field theory. The superposition structure can be constrained either by the microscopic causality in the bulk gravity or the bootstrap condition in the boundary conformal field theory. We show, contrary to some expectation, that these two conditions are not compatible to each other in the weak gravity regime. We also present an evidence to show that bulk local states in three dimensions are not organized by the Virasoro symmetry.

KEYwords: AdS-CFT Correspondence, Conformal Field Theory, Space-Time Symmetries ArXiv ePrint: 1605.00334 


\section{Contents}

$\begin{array}{llr}1 & \text { Introduction } & 1\end{array}$

2 Causality and cross-ratio 2

3 Microscopic causality in the bulk 4

$\begin{array}{lll}4 & \text { Bootstrap condition on crosscaps } & 7\end{array}$

$\begin{array}{llr}5 & \text { Gravity dual of crosscap states } & 8\end{array}$

6 Enhancement to the Virasoro symmetry 10

$\begin{array}{ll}6.1 \text { Virasoro enhancement at crosscaps } & 10\end{array}$

$\begin{array}{ll}\text { 6.2 No Virasoro enhancement for bulk local states } & 11\end{array}$

\section{Introduction}

In our previous paper [1], following [2,3], we pointed out that a bulk local state in a gravity theory in the anti-de Sitter space (AdS) is a linear superposition of Ishibashi states [4] for a crosscap in the dual conformal field theory (CFT). In this paper, we will discuss how to take the linear superposition.

Ishibashi's original construction is for boundary states, but they can be turned into crosscap states by applying the dilatation by the imaginary unit, corresponding to translation by one quarter of the period in the global Lorentzian time in AdS. For each primary state $|\phi\rangle$, one can define an Ishibashi state $|\phi\rangle\rangle$,

$$
\left.\left.M_{a b}|\phi\rangle\right\rangle=0, \quad\left(P_{a}+K_{a}\right)|\phi\rangle\right\rangle=0,
$$

preserving one half of the $\mathrm{SO}(2, d)$ global conformal symmetry of $\mathbb{R} \times S^{d-1}$, generated by the Hamiltonian $H$ along $\mathbb{R}$, the rotation $M_{a b}$ of $S^{d-1}$, translation $P_{a}$ and special conformal transformation $K_{a}(a=1, \ldots, d)$. The equations (1.1) were solved explicitly in [1] as,

$$
|\phi\rangle\rangle=\Gamma\left(\Delta-\frac{d}{2}+1\right)\left(\frac{P}{2}\right)^{d / 2-\Delta} J_{\Delta-d / 2}(P)|\phi\rangle,
$$

where $J_{\Delta-d / 2}(P)$ is the Bessel function of the first kind with $\Delta$ being the scaling dimension of $\phi$. It was observed in [1] that the dependence on the momentum $P$ is the same as that for the bulk-boundary smearing function in AdS, where the bulk point is evaluated at the center of $\mathrm{AdS}_{d+1}$ [5-7]. (For simplicity, we are discussing Ishibashi states for scalar primaries. See [1] for conditions when primary states carry non-zero spins.) 
The question we would like to address is how to take a linear superposition of Ishibashi states $|\phi\rangle\rangle$ over primary states $|\phi\rangle$ to construct a local state in the bulk AdS. One may be tempted to speculate that a bulk local state also has a special role to play in the dual CFT. A natural guess would then be that it satisfies consistency conditions for a crosscap in CFT, in particular a bootstrap condition for crossing symmetry, which are analogous to the Cardy conditions on boundary states.

We will show, contrary to such an expectation, that the bootstrap condition in CFT contradicts with the microscopic causality in AdS, which has been proposed as conditions on bulk local states in [8-12]. Namely, crosscap states obeying the bootstrap constraints generically do not correspond to local states in the bulk. We will also discuss bulk interpretation of crosscap states, which satisfy the bootstrap condition, and compare it with bulk local states satisfying the microscopic causality in AdS.

When $d=2$, the conformal symmetry is enhanced to the Virasoro symmetry. We will argue that a crosscap state in CFT preserves one half of the Virasoro symmetry, generalizing (1.1) to,

$$
\left.\left(L_{n}-(-1)^{n} \bar{L}_{-n}\right)|\phi\rangle\right\rangle_{\text {Virasoro }}=0,
$$

for the left and right Virasoro generators, $L_{n}, \bar{L}_{n}(n \in \mathbb{Z})$. On the other hand, we will present an evidence to show that the microscopic causality in AdS cannot be satisfied by a linear superposition of Ishibashi states of the Virasoro symmetry obeying (1.3). This also highlights the difference between local states in the bulk and crosscap states on the boundary.

It would be desirable to understand how to characterize bulk local states in the language of CFT. Our result shows that the bootstrap condition does not give a proper characterization of such states and that the Virasoro symmetry in two dimensions does not give a useful guiding principle to solve the microscopic causality in AdS.

This paper is organized as follows. In section II, we will review relations between the bulk and boundary coordinates and discuss causality and crossing symmetry in these coordinates. In section III, we discuss the microscopic causality conditions for local states in AdS and study solutions to these conditions. In section IV, we discuss the bootstrap condition on crosscaps in CFT, and compare their solutions to those of the microscopic causality conditions. In section $\mathrm{V}$, we discuss a bulk interpretation of crosscap states. This also highlights the difference between crosscap states in CFT and local states in AdS. In section VI, we discuss whether crosscap states and bulk local states can be organized usefully in the $\mathrm{AdS}_{3} / \mathrm{CFT}_{2}$ case by Ishibashi states of the Virasoro symmetry. We find that the answer is yes for crosscaps but no for bulk local states.

\section{Causality and cross-ratio}

A crosscap state can be used to compute correlation functions of CFT on the real projective plane, which is usually considered in the Euclidean signature. On the other hand, the causality in AdS should be discussed in the Lorentzian signature. Thus, in order to compare the bootstrap condition on the projective plane and the microscopic causality in AdS, it is 
useful to understand analytic continuation between coordinates. In this paper, we will use the global coordinates $(t, \rho, \Omega)$ of AdS with the metric,

$$
d s^{2}=-\cosh ^{2} \rho d t^{2}+d \rho^{2}+\sinh ^{2} \rho d \Omega^{2},
$$

where coordinates on $S^{d-1}$ are denoted by $\Omega$, which is identified with a unit vector in $\mathbb{R}^{d}$. As a consequence of working in the global patch, the causal interpretation of the crosscap cross-ratio $\eta$, defined below, is slightly different from that discussed in [8-12] in the Poincaré patch. We work in the global patch as we find it more convenient to compare the microscopic causality and the condition for the crosscap bootstrap.

In the Euclidean signature, the global coordinates with the metric,

$$
d s^{2}=\cosh ^{2} \rho d \tau^{2}+\sinh ^{2} \rho d \Omega^{2}+d \rho^{2},
$$

and the Poincaré coordinates with the metric,

$$
d s^{2}=\frac{d z^{2}}{z^{2}}+\frac{d x^{2}}{z^{2}}
$$

with $x \in \mathbb{R}^{d}$ cover the same $(d+1)$-dimensional hyperbolic space. In particular the center of Euclidean AdS $\tau=0, \rho=0$ corresponds to $z=1, x=0$.

On the boundary, the two coordinates are related to each other by the standard formula for the radial quantization, $x=e^{\tau} \Omega$. Thus, the involution,

$$
x \rightarrow \frac{x}{x^{2}},
$$

to define the real projective plane is,

$$
(\tau, \Omega) \rightarrow(-\tau,-\Omega)
$$

In the following, an important role is played by the crosscap cross-ratio $\eta$ of two points $x_{1}$ and $x_{2}$ on the plane defined by,

$$
\eta=\frac{\left(x_{1}-x_{2}\right)^{2}}{\left(1+x_{1}^{2}\right)\left(1+x_{2}^{2}\right)} .
$$

In the Euclidean signature, we always have $0 \leq \eta \leq 1$, and $\eta=1$ corresponds to the limit in which $x_{1}$ approaches the image of $x_{2}$, namely $x_{1} \rightarrow-x_{2} / x_{2}^{2}$. To see this, we note that $\eta \leq 1$ is equivalent to,

$$
1+2 x_{1} \cdot x_{2}+x_{1}^{2} x_{2}^{2}=x_{2}^{2}\left(\frac{x_{2}}{x_{2}^{2}}+x_{1}\right)^{2} \geq 0 .
$$

In the global coordinates (2.2), the cross-ratio is expressed as,

$$
\eta=\frac{\cosh \left(\tau_{1}-\tau_{2}\right)-\Omega_{1} \cdot \Omega_{2}}{\cosh \left(\tau_{1}-\tau_{2}\right)+\cosh \left(\tau_{1}+\tau_{2}\right)},
$$

Its Lorentzian continuation, $\tau=i t$, gives,

$$
\eta=\frac{\cos \left(t_{1}-t_{2}\right)-\Omega_{1} \cdot \Omega_{2}}{\cos \left(t_{1}-t_{2}\right)+\cos \left(t_{1}+t_{2}\right)},
$$


and takes values in $-\infty \leq \eta \leq \infty$. In the Lorentzian case, $\eta=1$ corresponds to the limit where $\left(t_{1}, \Omega_{1}\right)$ and $\left(-t_{2},-\Omega_{2}\right)$ are light-like separated.

Let us discuss a bulk interpretation of $\eta$ and relate it to the causality. Since the future light-cone from the center $(t=0, \rho=0)$ of AdS reaches the boundary at $t=\pi / 2$, a boundary point $(t, \Omega)$ is space-like separated from the center if and only if $|t|<\pi / 2$. Using this fact, we can show that, when $\eta>1$, at least one pair of the three points are space-like separated, modulo the $2 \pi$ period in $t$.

\section{Microscopic causality in the bulk}

A bulk local operator $\hat{\psi}$ is a function (more generally a section) over the bulk AdS and acts on the Hilbert space of the dual CFT. In [1], we required the action of the bulk isometry on $\hat{\psi}$ to be compatible with that of the corresponding conformal symmetry in the CFT,

$$
[J, \hat{\psi}]=i \mathcal{L}_{\mathcal{J}} \hat{\psi}
$$

where $\mathcal{J}$ is a Killing vector of the AdS corresponding to any one of the conformal generators, $H, M_{a b}, P_{a}, K_{a}$, and $\mathcal{L}_{\mathcal{J}}$ is the Lie derivative on $\hat{\psi}$ with respect to $\mathcal{J}$. Since the isotropy subgroup $\mathrm{SO}(1, d)$ at the origin $(t=0, \rho=0)$ is generated by $M_{a b}$ and $P_{a}+K_{a}$, the bulk local operator there should commute with them as,

$$
\begin{aligned}
{\left[M_{a b}, \hat{\psi}(0)\right] } & =0, \\
{\left[P_{a}+K_{a}, \hat{\psi}(0)\right] } & =0 .
\end{aligned}
$$

Correspondingly, the state $|\psi(0)\rangle=\hat{\psi}(0)|0\rangle$ satisfies the condition,

$$
\begin{aligned}
M_{a b}|\psi(0)\rangle & =0, \\
\left(P_{a}+K_{a}\right)|\psi(0)\rangle & =0,
\end{aligned}
$$

which we identified in [1] as a condition for crosscap states in CFT. Since Ishibashi states span the space of solutions to these equations, each bulk local state $|\psi(0)\rangle$ should be their linear superposition as,

$$
\left.|\psi(0)\rangle=\sum_{\phi} \psi_{\phi}|\phi\rangle\right\rangle
$$

The crosscap Ishibashi states may be regarded as a time-evolution of the boundary Ishibashi states by quarter period of the global Lorentzian time in AdS.

If $\hat{\psi}(t, \rho, \Omega)$ represents a single particle excitation in the bulk, it should approach a single trace primary operator $\phi_{0}(t, \Omega)$ at the boundary. Thus, $\psi_{\phi_{0}}=1$ in (3.4) and all other $\phi$ in the sum should have scaling dimensions larger than that of $\phi_{0}$. If only $\left.\left|\phi_{0}\right\rangle\right\rangle$ is in the sum, $\hat{\psi}$ would satisfy a free field equation in the bulk, because the crosscap Ishibashi state is an eigenstate of the Casimir operator of the conformal symmetry, which is equal the Laplacian in AdS when acting on $\hat{\psi}$ by (3.1).

To go beyond the free field limit in the bulk, it was proposed in [8-12] to impose the microscopic causality:

$$
[\hat{\psi}(X), \hat{\psi}(Y)]=0
$$


when the two points, $X$ and $Y$ are space-like separated. It turns out that $\psi_{\phi}$ in the expansion (3.4) can be determined order by order in the large $N$ expansion, as demonstrated to order $1 / N^{2}$ in $[12]$.

The first non-trivial constraint coming from the microscopic causality is of three-point functions (two on the boundary and one in the bulk). In the large $N$ limit, a bulk local state is equal to a particular Ishibashi state, and the three-point function can be expressed as a two-point functions evaluated on the Ishibashi state. To write down the three-point function, it is convenient to use the Poincaré coordinates $(z, x)$ with the metric,

$$
d s^{2}=\frac{d z^{2}+d x^{2}}{z^{2}}
$$

As shown in the previous section, the center of AdS in the global coordinates corresponds to $(z=1, x=0)$, which is where the bulk point is evaluated in the smearing function (1.2).

The two-point function for primary fields, $\phi_{1}$ and $\phi_{2}$, at the boundary points $x_{1}, x_{2}$ evaluated on the Ishibashi state $\left.\left|\phi_{3}\right\rangle\right\rangle$ is given by,

$$
\left.\left\langle 0\left|\phi_{1}\left(x_{1}\right) \phi_{2}\left(x_{2}\right)\right| \phi_{3}\right\rangle\right\rangle=\frac{\left(1+x_{1}^{2}\right)^{\frac{\Delta_{2}-\Delta_{1}}{2}}\left(1+x_{2}^{2}\right)^{\frac{\Delta_{1}-\Delta_{2}}{2}}}{\left(x_{1}-x_{2}\right)^{\Delta_{1}+\Delta_{2}}} g_{123}(\eta),
$$

where $\Delta_{1}$ and $\Delta_{2}$ are scaling dimensions of the primary fields dual to $\hat{\psi}_{1}$ and $\hat{\psi}_{2}$, and

$$
\eta=\frac{\left(x_{1}-x_{2}\right)^{2}}{\left(1+x_{1}^{2}\right)\left(1+x_{2}^{2}\right)}
$$

is a cross-ratio invariant under the $\mathrm{SO}(1, d)$ preserved by the crosscap. The function $g_{123}(\eta)$ takes the form,

$$
g_{123}(\eta)=C_{123} \eta^{\Delta_{3} / 2} \times{ }_{2} F_{1}\left(\frac{\Delta_{1}-\Delta_{2}+\Delta_{3}}{2}, \frac{\Delta_{2}-\Delta_{1}+\Delta_{3}}{2} ; \Delta_{3}+1-\frac{d}{2} ; \eta\right)
$$

where $C_{123}$ is the OPE coefficient of $\phi_{1}$ and $\phi_{2}$ into $\phi_{3}$.

One way to derive (3.9) is to use the explicit form of the scalar OPE with conformal descendants [13],

$\phi_{1}\left(x_{1}\right) \phi_{2}\left(x_{2}\right)=\sum_{i} \frac{C_{12 i}}{\left(x_{1}-x_{2}\right)^{\Delta_{1}+\Delta_{2}-\Delta_{i}}} C^{\Delta_{i}, \Delta_{1}-\Delta_{2}}\left(x_{1}-x_{2}, \partial_{x_{2}}\right) \phi_{i}\left(x_{2}\right)+$ higher spin tensors,

where

$$
C^{a, b}(x, \partial)=\frac{1}{B\left(a_{+}, a_{-}\right)} \int_{0}^{1} d \alpha \alpha^{a_{+}-1}(1-\alpha)^{a_{-}-1} \times \sum_{m=0} \frac{\left(-\frac{1}{4} x^{2} \alpha(1-\alpha) \partial^{2}\right)^{m}}{m !\left(a+1-\frac{1}{2} d\right)_{m}} e^{\alpha x \cdot \partial},
$$

with $a_{ \pm}=a \pm b$, and evaluate the one-point functions with the Ishibashi state, $\left.\left|\phi_{i}\right\rangle\right\rangle$.

Alternatively, one may act the conformal Casimir on the two-point function and solve the eigenvalue problem in the OPE limit as a boundary condition of the second order differential equation. The latter approach is more or less equivalent to solving the KleinGordon equation in the AdS space-time from the holographic perspective [9, 10]. The 
three-point function computed in this prescription is the Wightman function, so the nonzero commutator outside of the lightcone generates as a cut in $\eta$ when any two of the three-points are light-like separated.

The function $g_{123}(\eta)$ for generic values of $\Delta_{1,2,3}$ has a cut in $\eta>1$. As we saw in the previous section, when $\eta>1$, at least one pair of the three points becomes space-like separated. Therefore, it was proposed in [11] that the cut in $\eta>1$ should be cancelled by superposition of Ishibashi state and that this procedure determines the superposition coefficient $\psi_{\phi}$ order by order in the $1 / N$ expansions.

Let us illustrate the microscopic causality in AdS, by the following two examples. First we consider a free scalar field $\hat{\psi}$ in AdS. The three-point function of two $\hat{\psi}$ 's on the boundary and one composite operator $\hat{\psi} \times \hat{\psi}$ at the center of AdS, $z=1, x=0$, can be computed using the bulk boundary propagator,

$$
\langle\hat{\psi}(z=0, x) \hat{\psi}(z=1, x=0)\rangle=\left(\frac{1}{1+x^{2}}\right)^{\Delta}
$$

as,

$$
\left\langle\hat{\psi}\left(z=0, x_{1}\right) \hat{\psi}\left(z=0, x_{2}\right)[\hat{\psi} \times \hat{\psi}](z=1, x=0)\right\rangle_{\mathrm{AdS}}=\frac{2}{\left(1+x_{1}^{2}\right)^{\Delta}\left(1+x_{2}^{2}\right)^{\Delta}} .
$$

Setting the right-hand side to be equal to $G(\eta) /\left(x_{1}-x_{2}\right)^{2 \Delta}$, we find,

$$
G(\eta)=2 \eta^{\Delta}
$$

which does not have singularity or cut at $\eta=1$, as expected.

As another example, consider a local CFT in the bulk AdS (for holographic interpretation of such a model, see [14]). The three-point function can be computed using the conformal mapping from the flat space to AdS as,

$$
\left\langle\psi_{1}\left(z=0, x_{1}\right) \psi_{2}\left(z=0, x_{2}\right) \psi_{3}(z=1, x=0)\right\rangle_{\mathrm{AdS}}=\frac{\left(1+x_{1}^{2}\right)^{\frac{\Delta_{2}-\Delta_{1}}{2}}\left(1+x_{2}^{2}\right)^{\frac{\Delta_{1}-\Delta_{2}}{2}}}{\left(x_{1}-x_{2}\right)^{\Delta_{1}+\Delta_{2}}} G(\eta)
$$

with

$$
G(\eta)=C_{123} \eta^{\frac{\Delta_{3}}{2}}
$$

Again we find no singularity or cut at $\eta=1$.

In this case, we can compute the coefficient $\psi_{\phi}$ of the Ishibashi state expansion (3.4) of $|\hat{\psi}\rangle$ decomposition. Assuming $\Delta_{1}=\Delta_{2}$ for simplicity, we can expand $G(\eta)$ as

$$
\eta^{\frac{\Delta_{3}}{2}}=\sum_{n=0} C_{n} \eta^{\frac{\Delta_{3}}{2}+n} \times{ }_{2} F_{1}\left(\frac{\Delta_{3}}{2}+n, \frac{\Delta_{3}}{2}+n ; \Delta_{3}+2 n+1-\frac{d}{2} ; \eta\right) .
$$

with

$$
C_{n}=\prod_{k=1}^{n} \frac{\left(\Delta_{3}+2 k-2\right)^{2}}{2 k\left(d-2\left(\Delta_{3}+n+k-1\right)\right)}
$$

Therefore, we need to add the infinite tower of Ishibashi states with even integer spacing to reproduce this bulk operator $\psi$. Note that the coefficients are not $1 / N$ suppressed because the bulk theory is strongly interacting. Note also that not all the CFTs have such a structure of the operator spectrum. 


\section{Bootstrap condition on crosscaps}

In this section, we consider CFT on a $d$-dimensional real projective plane $\mathbb{R P}_{d}$, defined by quotienting the flat Euclidean space $\mathbb{R}^{d}$ by the involution,

$$
x \rightarrow-\frac{x}{x^{2}},
$$

which preserves the $\mathrm{SO}(1, d)$ subgroup of the Euclidean conformal symmetry $\mathrm{SO}(1, d+1)$. The fundamental domain may be taken as $x^{2} \geq 1$.

Conformally mapping the Euclidean space to the cylinder $\mathbb{R} \times S^{d-1}$, the involution (4.1) becomes $(\tau, \Omega) \rightarrow(-\tau,-\Omega)$, where $\tau$ is a coordinate on $\mathbb{R}$, and $\Omega$ is a unit vector in $\mathbb{R}^{d}$ parametrizing $S^{d-1}$. Analytically continuing to the Lorentzian signature cylinder, $t=-i \tau$, the involution becomes $(t, \Omega) \rightarrow(-t,-\Omega)$ and the fundamental domain may be taken as $t \geq 0$. If there is an additional global symmetry in CFT, the involution can be combined with $\phi \rightarrow \epsilon \phi$, where $\epsilon$ is taken as a $\mathbb{Z}_{2}$ element of the symmetry so that the action is compatible with the OPE.

Correlation functions of CFT on the real projective plane can be computed by using the crosscap state, which is a superposition of the Ishibashi states (1.2) as,

$$
\left.|C\rangle=\sum_{\phi} A_{\phi}|\phi\rangle\right\rangle
$$

The coefficient $A_{\phi}$ is related to the one-point function of a primary operator $\phi$ on the projective plane:

$$
\langle\phi(x)\rangle_{\mathbb{R P}_{d}}=\frac{A_{\phi}}{\left(1+x^{2}\right)^{\Delta_{\phi}}},
$$

where $\Delta_{\phi}$ is the scaling dimension of $\phi$. As noted $\mathrm{n}$ [1], the rotational invariance demands that only scalar operators have non-zero one-point functions on $\mathbb{R} \mathbb{P}_{d}$.

The two-point function of two scalar primary operators, $\phi_{1}\left(x_{1}\right)$ and $\phi_{2}\left(x_{2}\right)$, can be expressed as,

$$
\left\langle\phi_{1}\left(x_{1}\right) \phi_{2}\left(x_{2}\right)\right\rangle=\frac{\left(1+x_{1}^{2}\right)^{\frac{\Delta_{2}-\Delta_{1}}{2}}\left(1+x_{2}^{2}\right)^{\frac{\Delta_{1}-\Delta_{2}}{2}}}{\left(x_{1}-x_{2}\right)^{\Delta_{1}+\Delta_{2}}} G(\eta)
$$

and $G(\eta)$ has the conformal partial wave decomposition as,

$$
G(\eta)=\sum_{\phi} C_{12 \phi} A_{\phi} \eta^{\frac{\Delta_{\phi}}{2}} \times{ }_{2} F_{1}\left(\frac{\Delta_{1}-\Delta_{2}+\Delta_{\phi}}{2}, \frac{-\Delta_{1}+\Delta_{2}+\Delta_{\phi}}{2} ; \Delta_{\phi}+1-\frac{d}{2} ; \eta\right) .
$$

Consistency of CFT on $\mathbb{R P}_{d}$ requires the crossing symmetry of two-point functions [15, 16]. It compares the expansion (4.5) at $\eta=0$ to another expansion at $\eta=1$, where $x_{1}$ approaches the mirror image of $x_{2}$. Since the OPE is convergent and the two-point functions are analytic, we obtain the crossing equation or crosscap conformal bootstrap equation, [16],

$$
G(\eta)=\epsilon\left(\frac{\eta}{1-\eta}\right)^{\frac{\Delta_{1}+\Delta_{2}}{2}} G(1-\eta)
$$

where the possibility of non-trivial involution $\epsilon$ was first introduced in [17]. 
Clearly, both examples we discussed at the end of the last section - the free massless scalar field and the local CFT in AdS, where $G(\eta)=C_{123} \eta^{\frac{\Delta_{3}}{2}}$ - do not satisfy (4.6). This already shows a tension between the microscopic causality and the bootstrap condition.

To see that the bootstrap equation (4.6) is incompatible with the bulk locality in general, we can apply the conformal partial wave decomposition to the right-hand side of (4.6) as,

$$
\begin{aligned}
G(\eta)= & \epsilon\left(\frac{\eta}{1-\eta}\right)^{\frac{\Delta_{1}+\Delta_{2}}{2}} G(1-\eta) \\
= & \epsilon \eta^{\frac{\Delta_{1}+\Delta_{2}}{2}} \sum_{\phi} C_{12 \phi} A_{\phi}(1-\eta)^{\frac{\Delta_{\phi}-\Delta_{1}-\Delta_{2}}{2}} \\
& \times{ }_{2} F_{1}\left(\frac{\Delta_{1}-\Delta_{2}+\Delta_{\phi}}{2}, \frac{-\Delta_{1}+\Delta_{2}+\Delta_{\phi}}{2} ; \Delta_{\phi}+1-\frac{d}{2} ; 1-\eta\right) .
\end{aligned}
$$

We see that $G(\eta)$ contains a cut at $\eta>1$ because of the factor of $(1-\eta)^{\frac{\Delta_{\phi}-\Delta_{1}-\Delta_{2}}{2}}$ if $\Delta_{\phi}-\Delta_{1}-\Delta_{2}$ is not an even integer. We conclude that a solution to the bootstrap equation (4.6) cannot satisfy the microscopic causality, unless $C_{12 \phi} A_{\phi}=0$ for all $\phi$ 's with $\Delta_{\phi} \notin \Delta_{1}+\Delta_{2}+2 \mathbb{Z}$.

It may also be instructive to examine a simple solution to the crosscap bootstrap equation, given by a free scalar field $\phi(x)$ in $d$-dimension with $\Delta_{\phi}=\frac{d}{2}-1$, and see if they satisfy the microscopic causality. By using the method of image, the two-point function on $\mathbb{R P}_{d}$ can be computed as

$$
\left\langle\phi\left(x_{1}\right) \phi\left(x_{2}\right)\right\rangle_{\mathbb{R P}_{d}}=\frac{1}{\left(x_{1}-x_{2}\right)^{d-2}}+\frac{\epsilon}{\left(1+2 x_{1} \cdot x_{2}+x_{1}^{2} x_{2}^{2}\right)^{\frac{d}{2}-1}},
$$

where $\epsilon= \pm 1$ reflects the additional $\mathbb{Z}_{2}$ symmetry on the free scalar field $\phi \rightarrow \pm \phi$, which can be combined with the involution. The corresponding $G(\eta)$ is given by,

$$
G(\eta)=1+\epsilon\left(\frac{\eta}{1-\eta}\right)^{\frac{d}{2}-1}
$$

This satisfies the conformal bootstrap equation, but the microscopic causality is violated when $d$ is not even.

\section{$5 \quad$ Gravity dual of crosscap states}

We found that the microscopic causality for local states in AdS and the bootstrap condition for crosscap states in CFT are generically not compatible to each other. Given this, one may ask if crosscap states have a different geometric interpretation in AdS. In this section, we discuss a straightforward interpretation using an involution on AdS and find that its properties are different from those expected for bulk local states.

On the fixed AdS background, the involution acts on the global coordinates

$$
d s^{2}=-\cosh \rho^{2} d t^{2}+d \rho^{2}+\sinh \rho^{2} d \Omega^{2},
$$


as,

$$
(t, \rho, \Omega) \rightarrow(-t, \rho,-\Omega) .
$$

The involution preserves the $\mathrm{SO}(2, d-1)$ subgroup of the AdS isometry, and at the boundary it reduces to the field theory involution discussed in the previous section. The bulk fields are identified as

$$
\hat{\psi}(t, \rho, \Omega) \rightarrow \epsilon \hat{\psi}(-t, \rho,-\Omega),
$$

where we are allowing a possibility of an additional $\mathbb{Z}_{2}$ action $\epsilon$ on $\hat{\psi}$.

After the Euclidean continuation, both the global coordinates $(\tau, \rho, \Omega)$ and the Poincaré coordinates $(z, x)$ cover the entire hyperbolic space. Therefore, the Euclidean continuation of the involution (5.3) can be expressed in the Poincaré coordinates as,

$$
(z, x) \rightarrow\left(\frac{z}{z^{2}+x^{2}}, \frac{-x}{z^{2}+x^{2}}\right)
$$

The fundamental domain can be taken $z^{2}+x^{2} \geq 1$.

The crosscap state $|C\rangle$ defined in this way is a superposition of the Ishibashi states,

$$
\left.|C\rangle=\sum_{\phi} A_{\phi}|\phi\rangle\right\rangle
$$

The coefficients $A_{\phi}$ 's are computable in the bulk as the one-point function of the bulk field $\hat{\psi}$ dual to $\phi$ on the boundary. The one-point function vanishes unless $\phi$ is scalar.

If the bulk gravity theory is weakly coupled, the bulk field $\hat{\psi}$ corresponding to a singletrace scalar operator $\phi$ can be described approximately by the free theory,

$$
S=\int d^{d+1} x \sqrt{-g}\left(\partial_{\mu} \hat{\psi} \partial^{\mu} \hat{\psi}+m^{2} \hat{\psi}^{2}\right) .
$$

In this case, the one-point function vanishes because of the $\mathbb{Z}_{2}$ symmetry of the action under $\psi \rightarrow-\psi$. We therefore predict that, for all single trace operators, $A_{\phi}=0$ in the weakly coupled gravity regime. Note that this argument does not apply to multi-trace operators since composites of even number of $\hat{\psi}$ 's are $\mathbb{Z}_{2}$ even.

Continuing to work in the weakly coupled gravity limit, two-point function of single trace operators $\phi_{1}$ and $\phi_{2}$ can be computed using the method of image as,

$$
G(\eta)=\delta_{\phi_{1}, \phi_{2}}\left(1+\epsilon\left(\frac{\eta}{1-\eta}\right)^{\Delta_{\phi_{1}}}\right),
$$

with the choice of the involution $\epsilon= \pm 1$. This reproduces the two-point function of the generalized free field theory on the real projective plane and satisfies the crosscap bootstrap equation (4.6). The conformal partial wave decomposition of (5.7) generates infinite towers of double-trace operators as,

$$
G(\eta)=\sum_{n} C_{n} \eta^{\Delta_{\phi_{1}}+n} \times{ }_{2} F_{1}\left(\Delta_{\phi_{1}}+n, \Delta_{\phi_{1}}+n ; 2 \Delta_{\phi_{1}}+2 n+1-\frac{d}{2} ; \eta\right),
$$


with $C_{0}=1, C_{1}=\epsilon \Delta_{1}, C_{2}=\epsilon \frac{(-2+d) \Delta_{1}\left(1+\Delta_{1}\right)}{2\left(-6+d-4 \Delta_{1}\right)}$ and so on. Therefore the bulk crosscap state $|C\rangle$ contains the corresponding infinite towers of Ishibashi states for the double trace operators of the form, $\hat{\psi} \square^{n} \hat{\psi}$ (This infinite sum can be truncated in the free massless scalar case since $\square \hat{\psi}=0$ ). This can be repeated for two-point functions of multi-trace operators to show that the crosscap state $|C\rangle$ contains an infinite tower of multi-trace Ishibashi states as well.

We have found that contributions of Ishibashi states for single-trace operators are suppressed in crosscap states in the weakly coupled gravity limit due to the $\mathbb{Z}_{2}$ symmetry $\hat{\psi} \rightarrow-\hat{\psi}$ of the free scalar action (5.6). In contrast, bulk local states are dominated by single-trace states in the same limit. This also highlights the difference between crosscap states and bulk local states.

Recently it was suggested in [18] that CFT on the two-dimensional projective plane may not have a smooth geometric dual. This may be related to the fact the bulk involution (5.2) has a fixed point at the origin of AdS and quotieting by it may generate an orbifold singularity in the bulk.

\section{Enhancement to the Virasoro symmetry}

When $d=2$, the global conformal symmetry is enhanced to the Virasoro symmetry. We will argue that crosscap states preserve one half of the full Virasoro symmetry in this case and that we can use Ishibashi states for the full Virasoro symmetry rather than the global conformal symmetry to expand crosscap states. On the other hand, we will provide some evidence that bulk local states are not necessarily organized by the Virasoro symmetry.

\subsection{Virasoro enhancement at crosscaps}

Before discussing crosscap states, it would instructive to review the case for boundary states. Consider a $(t, \sigma)$ plane and place a boundary located at $t=0$ and extending in the $\sigma$-direction. Because of the scale invariance, the left and right-moving components of the energy-momentum tensor match at the boundary up to a total derivative along the boundary as,

$$
T_{\sigma t}(t=0, \sigma)=T(t=0, \sigma)-\bar{T}(t=0, \sigma)=\partial_{\sigma} j_{\sigma \sigma}(\sigma) .
$$

In addition, if we require the local Weyl invariance on the boundary, the total derivative term must vanish $\partial_{\sigma} j_{\sigma \sigma}=0$ and that the boundary preserves half of the bulk Virasoro symmetry [19].

There is one more possibility: if we only require the global conformal invariance at the boundary, the condition becomes [20],

$$
j_{\sigma \sigma}(\sigma)=\partial_{\sigma} \ell_{\sigma}(\sigma)
$$

If this is non-zero, the boundary preserves one half of the global conformal symmetry but not of the full Virasoro symmetry. The reason why such a possibility exists at all is because we can always put an additional $0+1$ dimensional conformal quantum mechanical system at the boundary, which does not necessarily have the Virasoro symmetry. 
The situation is different for crosscap states, where we cannot introduce localized degrees of freedom. In particular, if the involution we used to define a crosscap acts trivially on the energy-momentum tensor, the global conformal invariance alone demands that the crosscap condition takes the form,

$$
T(t, \sigma)-\bar{T}(-t, \sigma+\pi)=0 .
$$

In this case, one half of the full Virasoro symmetry is automatically preserved.

One consequence of this is that the bootstrap condition with the Virasoro symmetry is the same as the one with only the global conformal symmetry. Indeed, the numerical analysis in [16] shows that, in simple models such as the $2 d$ critical Ising model, the bootstrap condition for the crosscap is so strong that the solution automatically respects the Virasoro symmetry.

There is one caveat: when the energy-momentum tensor is a part of a larger chiral algebra such as the W-symmetry, there is a possibility to introduce non-trivial action on $T_{\mu \nu}$ under the involution. From the holographic viewpoint, this can happen in higher spin theories.

Preserving one-half of the Virasoro symmetry imposes strong constraints on solutions to the bootstrap equation. In fact, the constraints can be too strong to have any solution at all. For example, a heterotic CFT with different values of Virasoro central charges for its left and right-movers do not admit an involution on the real projective plane.

\subsection{No Virasoro enhancement for bulk local states}

Let us turn to the microscopic causality conditions. We will use the $2 d$ critical Ising model as an example to see if the conditions can be satisfied by a superposition of Ishibashi states for the full Virasoro symmetry.

In the critical Ising model, the Virasoro OPE gives,

$$
[\sigma] \times[\sigma]=[1]+[\epsilon]
$$

and because of the Virasoro symmetry, one may construct the crosscap state from the Virasoro Ishibashi states as,

$$
\left.|C\rangle=|1\rangle\rangle_{\text {Virasoro }}+\frac{\sqrt{2}-1}{2}|\epsilon\rangle\right\rangle_{\text {Virasoro }} .
$$

Correspondingly, the two-point function of the spin operator $\sigma$ on the real projective plane can be decomposed as,

$$
G_{\sigma \sigma}(\eta)=(1-\eta)^{3 / 8}{ }_{2} F_{1}\left(\frac{3}{4}, \frac{1}{4} ; \frac{1}{2} ; \eta\right)+\frac{\sqrt{2}-1}{2} \eta^{1 / 2}(1-\eta)^{3 / 8}{ }_{2} F_{1}\left(\frac{3}{4}, \frac{5}{4} ; \frac{3}{2} ; \eta\right) .
$$

This two-point function satisfies the bootstrap equation for the crossing symmetry, $G(\eta)=$ $[\eta /(1-\eta)]^{\Delta_{\sigma}} G(1-\eta)$.

Let us turn our attention to the microscopic causality condition. The question is whether it is possible to take an appropriate superposition,

$$
G(\eta)=(1-\eta)^{3 / 8}{ }_{2} F_{1}\left(\frac{3}{4}, \frac{1}{4} ; \frac{1}{2} ; \eta\right)+\psi_{\epsilon} \eta^{1 / 2}(1-\eta)^{3 / 8}{ }_{2} F_{1}\left(\frac{3}{4}, \frac{5}{4} ; \frac{3}{2} ; \eta\right),
$$


to cancel the cut at $\eta>1$ by adjusting the parameter $\psi_{\epsilon}$. It turns out that it is not possible. Since both conformal blocks,

$$
\begin{aligned}
& { }_{2} F_{1}\left(\frac{3}{4}, \frac{5}{4} ; \frac{3}{2} ; \eta\right)=\sqrt{\frac{2}{\eta}} \cdot \frac{\sqrt{1-\sqrt{1-\eta}}}{\sqrt{1-\eta}} \\
& { }_{2} F_{1}\left(\frac{3}{4}, \frac{1}{4} ; \frac{1}{2} ; \eta\right)=\frac{1}{\sqrt{2}} \cdot \frac{\sqrt{1+\sqrt{1-\eta}}}{\sqrt{1-\eta}} .
\end{aligned}
$$

have cut for $\eta>1$ in both their denominators and numerators, it is not possible to cancel them by adjusting the single parameter $\psi_{\epsilon}$. In this case, we cannot construct a solution to the microscopic causality by a superposition of Ishibashi states for the Virasoro symmetry.

Though we do not expect that the $2 d$ Ising model has a weakly coupled gravity description, this illustrates the difficulty in cancelling cuts at $\eta>1$ by a superposition of Virasoro conformal blocks for a crosscap.

\section{Acknowledgments}

We thank Alex Maloney, Tadashi Takayanagi, and Herman Verlinde for discussions. Our research is supported in part by the World Premier International Research Center Initiative (WPI Initiative), MEXT, Japan. The research of HO is also supported in part by U.S. DOE grant DE-SC0011632, by the Simons Investigator Award, by Caltech's Walter Burke Institute for Theoretical Physics and Moore Center for Theoretical Cosmology and Physics, by JSPS Grant-in-Aid for Scientific Research C-26400240, and by JSPS Grant-in-Aid for Scientific Research on Innovative Areas 15H05895. HO thank the hospitality of the Institute for Advanced Study as Director's Visiting Professor in the fall 2015, where this work was initiated, and of the Center for Mathematical Sciences and Applications and the Center for the Fundamental Laws of Nature at Harvard University as a visiting scholar in the spring 2016, where this work was completed.

Open Access. This article is distributed under the terms of the Creative Commons Attribution License (CC-BY 4.0), which permits any use, distribution and reproduction in any medium, provided the original author(s) and source are credited.

\section{References}

[1] Y. Nakayama and H. Ooguri, Bulk locality and boundary creating operators, JHEP 10 (2015) 114 [arXiv:1507.04130] [INSPIRE].

[2] H. Verlinde, Poking holes in AdS/CFT: bulk fields from boundary states, arXiv:1505.05069 [INSPIRE].

[3] M. Miyaji, T. Numasawa, N. Shiba, T. Takayanagi and K. Watanabe, Continuous multiscale entanglement renormalization ansatz as holographic surface-state correspondence, Phys. Rev. Lett. 115 (2015) 171602 [arXiv:1506.01353] [INSPIRE].

[4] N. Ishibashi, The boundary and crosscap states in conformal field theories, Mod. Phys. Lett. A 4 (1989) 251 [INSPIRE]. 
[5] T. Banks, M.R. Douglas, G.T. Horowitz and E.J. Martinec, AdS dynamics from conformal field theory, hep-th/9808016 [INSPIRE].

[6] V. Balasubramanian, P. Kraus and A.E. Lawrence, Bulk versus boundary dynamics in anti-de Sitter space-time, Phys. Rev. D 59 (1999) 046003 [hep-th/9805171] [INSPIRE].

[7] I. Bena, On the construction of local fields in the bulk of $A d S_{5}$ and other spaces, Phys. Rev. D 62 (2000) 066007 [hep-th/9905186] [INSPIRE].

[8] A. Hamilton, D.N. Kabat, G. Lifschytz and D.A. Lowe, Holographic representation of local bulk operators, Phys. Rev. D 74 (2006) 066009 [hep-th/0606141] [INSPIRE].

[9] D. Kabat, G. Lifschytz and D.A. Lowe, Constructing local bulk observables in interacting AdS/CFT, Phys. Rev. D 83 (2011) 106009 [arXiv:1102.2910] [INSPIRE].

[10] D. Kabat and G. Lifschytz, Decoding the hologram: scalar fields interacting with gravity, Phys. Rev. D 89 (2014) 066010 [arXiv:1311.3020] [InSPIRE].

[11] D. Kabat and G. Lifschytz, Bulk equations of motion from CFT correlators, JHEP 09 (2015) 059 [arXiv: 1505.03755] [INSPIRE].

[12] D. Kabat and G. Lifschytz, Locality, bulk equations of motion and the conformal bootstrap, arXiv: 1603.06800 [INSPIRE].

[13] D.M. McAvity and H. Osborn, Conformal field theories near a boundary in general dimensions, Nucl. Phys. B 455 (1995) 522 [cond-mat/9505127] [INSPIRE].

[14] O. Aharony, M. Berkooz and S.-J. Rey, Rigid holography and six-dimensional $N=(2,0)$ theories on $A d S_{5} \times S^{1}$, JHEP 03 (2015) 121 [arXiv: 1501.02904] [INSPIRE].

[15] D. Fioravanti, G. Pradisi and A. Sagnotti, Sewing constraints and nonorientable open strings, Phys. Lett. B 321 (1994) 349 [hep-th/9311183] [INSPIRE].

[16] Y. Nakayama, Bootstrapping critical Ising model on three-dimensional real projective space, Phys. Rev. Lett. 116 (2016) 141602 [arXiv:1601.06851] [InSPIRE].

[17] G. Pradisi, A. Sagnotti and Y.S. Stanev, Planar duality in $\mathrm{SU}(2)$ WZW models, Phys. Lett. B 354 (1995) 279 [hep-th/9503207] [INSPIRE].

[18] A. Maloney and S.F. Ross, Holography on non-orientable surfaces, Class. Quant. Grav. 33 (2016) 185006 [arXiv:1603.04426] [INSPIRE].

[19] D.M. McAvity and H. Osborn, Energy momentum tensor in conformal field theories near a boundary, Nucl. Phys. B 406 (1993) 655 [hep-th/9302068] [INSPIRE].

[20] Y. Nakayama, Is boundary conformal in CFT?, Phys. Rev. D 87 (2013) 046005 [arXiv: 1210.6439] [INSPIRE]. 